\title{
Application of Computer Numerical Simulation in Movement Technique Research
}

\author{
Wen Jin 1 ,a \\ ${ }^{1}$ Sports Department of Zhengzhou Normal University, Zhengzhou, 450000, China \\ aemail: lylglg@163.com.
}

Key words: computer numerical simulation, movement technique, rigid model

\begin{abstract}
Adopt the literature review method, apply the computer numerical simulation technology, and combine the research method and characteristics of the physical exercise technology. It discusses the application methods, process, specific steps and function of the computer numerical simulation technique from the establishment of the sport technique simulation model, steps, data input, and data pre-processing of the computer numerical simulation, computer numerical simulation calculation, modification of the movement technique proposal, application of the new action design and computer numerical simulation in the movement technique training, etc.

In the physical exercise teaching and exercise training practice, it generally uses the camera to shoot the athletes' skilled movement, and then analyzes it. However, this method only can simply replay the skilled movement of the athletes, while its quantization and comparative analysis on the kinematics, dynamics, and biology of the movement technique cannot achieve satisfactory result. The rapid development of the modern computer technology lays scientific foundation for the comparison, superposition, dynamics test and calculation of the movement technique image. Especially, the computer numerical simulation technique enables the coach to predict the result of the movement technique in the design phase of the movement technique and then proceed with the optimal design of the movement technique. No matter for the improvement of the movement technique or the reconstruction of the movement technique, it is feasible to greatly shorten the research period, and improve the feasibility and actual application value of the movement technique design proposal.
\end{abstract}

\section{Establishment of the Digital Simulation Model of the Human Motion}

The system simulation is a powerful tool for using the computer to analyze and design various complex systems. Its application and influence have been widely applied in many science researches, engineering domain and non-engineering domain. In the movement technique research, as the research is involved in the features of the human motion, the human body is generally simplified into a multiple rigid system mode according to the purpose of the movement technique, rules and features of the human to finish the movement technique. The skeleton, muscle, tendon, and other movement system tissue of the human body is processed into the acting force and torque among each rigid by, and then the kinematics and dynamics equation for studying the human motion is established. When the independent generalized coordinate is adopted to study the skilled movement, its mathematical model is an ordinary differential equation[1]. If the dependent generalized coordinate is adopted, the differential algebra equation will be achieved. These equations generally belong to higher-order and highly-coupled non-linear equation. Apart from simplifying and achieving the analysis results under tiny minority of the situations, the arithmetic solution can be achieved through the computer in general. 


\section{Methods and Procedure of the Computer Numerical Simulation}

\subsection{Methods of computer numerical simulation}

The methods for simulating the human movement through the computer include: input the original parameter, calculate, achieve the description result of the skilled movement, form the principle of the movement technique, etc. This process is named as numerical simulation. During the calculation, it is possible to face positive and reverse problems. For instance, get the mechanical conditions (e.g. muscle moment force, drag torque, human hypogene transmission and angular momentum transmission, exogenous process, etc.) that form the skilled movement when the movement status (e.g. space and time feature of the movement of each link) of each link of the human body is known. During the computing progress of the computer numerical simulation, it is necessary to increase the mechanics additional constraint conditions, so as to get the optimal combination of each input amount, and finish the optimal design of the movement technique. Under the condition of establishing accurate parameters of the numerical simulation model, the calculation result can accurately reflect the actual movement status, which has strong prediction and practical guidance effect for the results obtained by the research on the movement technique.

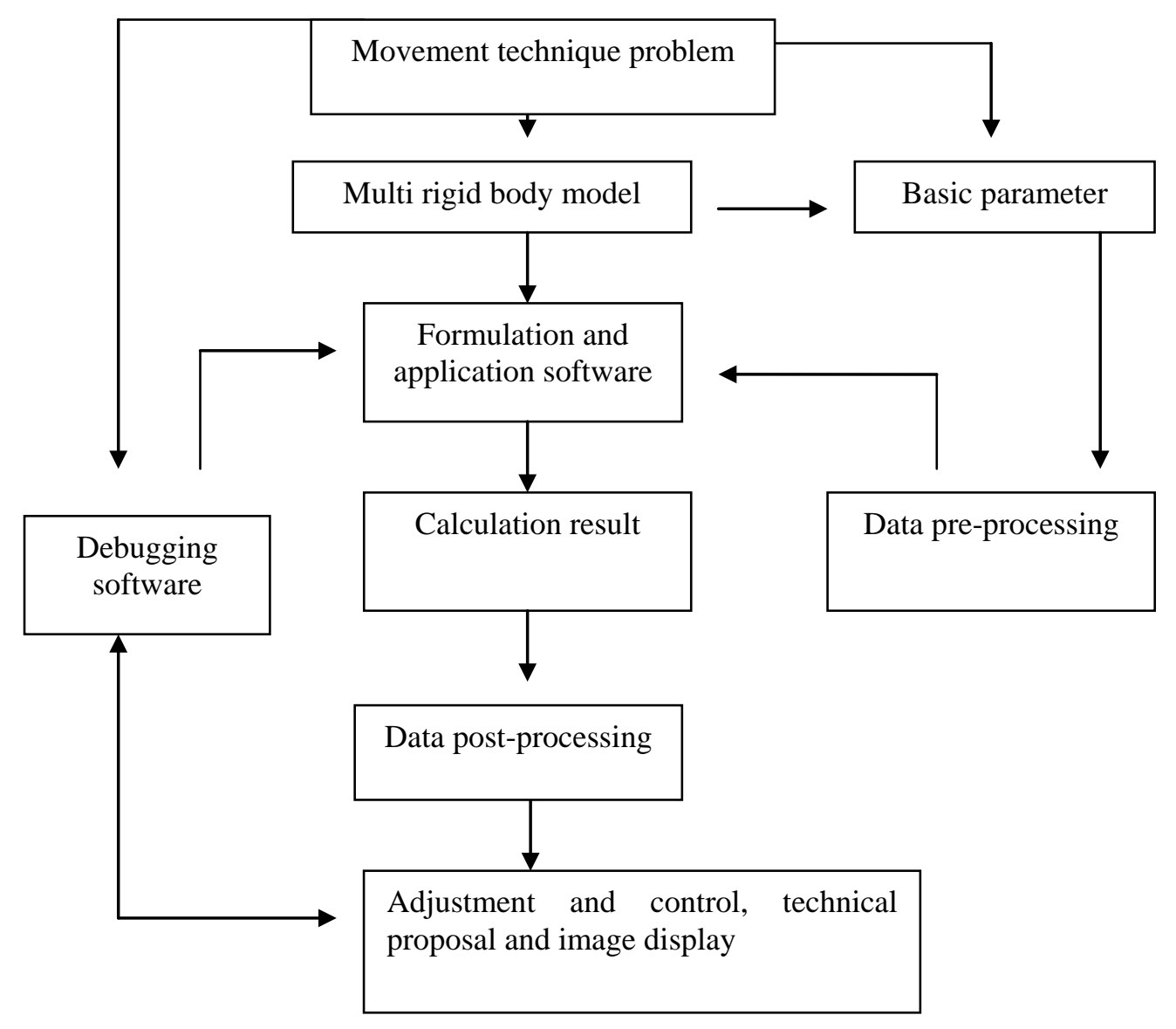

Picture 1 working process diagram of the computer numerical simulation

It puts forward the optimal movement technique proposal toward the throwing technique of Wilkinson. As this research is established on the basis of the scientific theory, it an provide scientific guidance for optimizing the movement technique of the athletes, which has great significance in improving the movement technique level[2].

2.2 Procedure of the computer numerical simulation

The procedure of the computer numerical simulation gets involved in different aspects. For instance, according to the researched movement technique subject, target and task, establish continuous movement digitalization model of the multiple rigid body system, establish the 
movement sports biomechanics equation, apply or compile the software, carry out the debugging calculation, data review and calibration, feedback adjustment and control, etc. The working process is shown in Picture 1.

\section{Steps of the Computer Numerical Simulation}

With the rapid development of the modern electronics computer technique, there already has been much computer program software for studying the rigid body system model dynamics. Through such software, it is feasible to solve the positive and reverse problems of the multiple rigid body system kinematics during the movement. As all movement of the human body must comply with the basic principles of the mechanics and biology, it is feasible to solve the movement technique problems of the human body during the sports through the calculation of the numerical simulation. The steps of the computer numerical simulation include data input, data pre-processing, simulation calculation, data post-processing, skilled movement proposal modification, or innovation movement design, data pre-processing, and image display.

\subsection{Data input}

The data input includes the 3D film material or kinematics parameter shot by the plane video, kinetic parameter, human body inertia, rotational inertia and other biomechanics obtained by the electronic ergograph system. The data input process has the statistical processing, the screening, modification and smoothing procedure of the input data. It can reconfirm and modify the incorrect or doubtful data[3].

\subsection{Data processing}

The data pre-processing include the coordinate switching, error processing, time synchronization processing of the data, etc. According to the film or video data, the reference coordinate (e.g. background reference marker, coordinates of the human body structure, etc.) with inertia and without Inertia is adopted to present the movement spatial feature. The coordinate system of the computer simulation calculation is generally established on the inertia of the multiple rigid body models. Therefore, before the computing, it is necessary to carry out coordinate conversion of the original data, make statistical processing on the parameters, eliminate the singular points on the error processing, and carry out smoothing processing on the whole set of the data. The time synchronization processing is the coinciding of the spatial data and time feature of the plane image, or synchronously modifying the image data of the planes that were vertical to each other in the 3D shooting. This data pre-processing has direct influence and effect on the accuracy of the system calculation result.

\subsection{Computer simulation computing}

After the input data goes through the correct pre-processing, it is feasible to obtain the whole process of the human body in the spatial movement and verify the accuracy of the simulation procedure through comparing with the actual image according to the kinetic equation. When the simulation result picture of the simulation computing is compared with the actual movement image, if the tracks of both are basically identical, it indicates that the computer numerical simulation procedure calculation is in line with the research reality. Its result has good guidance effect and significance on the improvement and reconstruction of the movement technology and the exercise training practice.

3.4 Modification of the human body movement and new action design

An important function of the computer numerical simulation computing technique is to modify some movement and design a new optimal movement technique proposal. As the image change can adopt the mathematical language description, the whole movement image will generate corresponding change after the data is input into the modification of some movement through the automatic identification of the computer system. If the results displayed in the computer is ideal, it indicates that to modify or design new movement is worthy of trying. Such function is helpful for the coach and athlete during the exercise training, especially the confirmation of the basic movement, creating difficulty and new movement, which will enable the coach's new envisage to be confirmed with feasibility and effectiveness. Then, the actual attempt is carried out, which can 
reduce and prevent the movement injury, and improve the feasibility and safety of the movement technique training.

3.5 Data post-processing and image display

The data obtained by the computer numerical simulation computing should be displayed in different ways according to the requirement of the research subject content and task[4]. The computer numerical simulation computing procedure has various data conversion functions, and it can display the results in different image ways. This procedure is called as data post-processing. Finally, it is feasible to obtain the most reasonable and intuitive skilled movement image results. In the process of studying and designing the optimal movement technique proposal, the continuous image output obtained through the computer numerical simulation computing has great reference value for the modification and innovation of the movement technique proposal. Moreover, it gives full play to the direct and decisive effect.

\section{Application Value of the Computer Numerical Simulation in the Movement Technique Training}

When carrying out data simulation through the computer and studying the skilled improvement through the animation research, it has the following function and effect role.

(1) Carry out the data simulation through the computer, adopt 3D vivid simulation technique movement, display the movement through the animation, and analyze the kinematics, dynamics and biological characteristics of the movement technique. Make the athletes to master the key points of the skilled movement more easily and rapidly, so as to greatly improve the athletes' overall exercise training level and movement skill levels[5].

(2) Simulate the movement arrangement, design reasonable movement system, and complete the design of the new movement technique proposal and standard skilled movement model. Through the computer numerical simulation technology, it is feasible to edit, modify and design new movement, select and design optimal movement technique proposal, establish movement technique mode, and guide the movement technique teaching and training.

(3) The comparison between the simulation result and training technique is convenient for the comparative analysis and technical diagnosis on the skilled movement, and displaying the analysis results through the image form. It includes the spatial status change of the human movement, change of the gravity tract, displacement, velocity, acceleration, force and moment, etc[6]. On that basis, it is feasible to make in-depth comparative analysis on the movement and athletes' skilled movement. Design the guidance proposal of the skilled movement through displaying the athletes' training movement and standard simulation movement on the same screen and carrying out the technical diagnosis with the same perspective and synchronous comparison according to the movement technique principle. Although the domestic and foreign peers have gained some results from the research on the computer numerical simulation computing technique of the human body movement simulation, it is necessary to set complex parameters by hands due to the high complexity of the computing, and it cannot fully meet the demands of the exercise practice. With the rapid development of the modern science and technology, the computer numerical simulation computing technique will present greater guidance effect and value during the constant practice with the movement technique research.

\section{References}

[1] Zheng Xiuyuan. Modern Sports Biomechanics [M]. Beijing: National Defence Industry Press, 2002.

[2] Fen Genquan. Ergonomics [M]. Lanzhou: Gansu People’s Publishing House, 1980.

[3] Li Liangbiao. Sports Biomechanics [M]. Beijing: Beijing Institute of Physical education Press, 1991.

[4] Shi Xuehuang. Sports Biomechanics Translation Set [M]. Beijing: Tsinghua University Press, 1985. 
[5] Wei Wenyi. Sports Biomechanics Translation Set [M]. Beijing: Tsinghua University Press, 1989.

[6] Wu Xuguang. Computer Simulation Technique [M]. Beijing: Chemical Industry Press, 2005. 